\title{
Philosophiques
}

\section{L’évolutionnisme économique de Friedrich Hayek}

\section{Robert Nadeau}

Volume 25, numéro 2, automne 1998

Les modèles d'évolution en économie et en sciences sociales

URI : https://id.erudit.org/iderudit/027490ar

DOI : https://doi.org/10.7202/027490ar

Aller au sommaire du numéro

Éditeur(s)

Société de philosophie du Québec

ISSN

0316-2923 (imprimé)

1492-1391 (numérique)

Découvrir la revue

Citer cet article

Nadeau, R. (1998). L'évolutionnisme économique de Friedrich Hayek.

Philosophiques, 25(2), 257-279. https://doi.org/10.7202/027490ar

\section{Résumé de l'article}

Une analyse minutieuse du dernier ouvrage de Hayek publié quatre ans avant sa mort, soit en 1988 (La Présomption fatale), permet de mettre au Jour les tenants et aboutissants de la perspective évolutionnaire épousée par Hayek en matière d'économie politique. Examinant d'abord le concept d'ordre spontané, exposant ensuite le fonctionnement de Véconomie marchande comme système en expansion continue, je montre alors comment s'articulent pour Hayek compétence économique et compétence épistémique d'un côté, puis coordination économique et compétence morale de l'autre. L'analyse fait apercevoir que, fondamentalement, l'optique de Hayek est évolutionnaire parce qu 'elle est résolument populationnelle, ce que les commentateurs de Hayek ont plutôt ignoré jusqu'ici, ce qui fournit l'occasion de passer en revue les critiques adressées par Hayek au principe de population de Malthus (1798).
Ce document est protégé par la loi sur le droit d'auteur. L'utilisation des services d’Érudit (y compris la reproduction) est assujettie à sa politique d'utilisation que vous pouvez consulter en ligne.

https://apropos.erudit.org/fr/usagers/politique-dutilisation/ 


\title{
L'ÉVOLUTIONNISME ÉCONOMIQUE dE RRIEDRICH HAYEK
}

\author{
PAR \\ Robert Nadeau
}

RÉSUMÉ : Une analyse minutieuse du dernier ouvrage de Hayek publié quatre ans avant sa mort, soit en 1988 (La Présomption fatale), permet de mettre au jour les tenants et aboutissants de la perspective évolutionnaire épousée par Hayek en matière d'économie politique. Examinant d'abord le concept d'ordre spontané, exposant ensuite le fonctionnement de l'économie marchande comme système en expansion continue, je montre alors comment s'articulent pour Hayek compétence économique et compétence épistémique d'un côté, puis coordination économique et compétence morale de l'autre. L'analyse fait apercevoir que, fondamentalement, l'optique de Hayek est évolutionnaire parce qu' elle est résolument populationnelle, ce que les commentateurs de Hayek ont plutôt ignoré jusqu'ici, ce qui fournit l'occasion de passer en revue les critiques adressées par Hayek au principe de population de Malthus (1798).

ABSTRACT: Four years before his death, Hayek published a book (The Fatal Conceit, 1988) in which he rearticulates the main reasons for adopting an evolutionary point of view in economics. This paper tries precisely to systematize these reasons as clearly as possible. I begin by a systematic discussion of the concept of spontaneous order. I then look at what mainly defines the catallaxy as a still extending market order. Following this line of theoretical argument, I then try to show how for Hayek economic competence and epistemic competence are to be seen as developing together in a free market economy. Furthermore, I try to show how for Hayek economic coordination and moral competence are to be seen as the unintended results of rule-governed individual dimensions of behaviour mutually

Je remercie le Fonds pour la formation de chercheurs et l'aide à la recherche du Québec (Fonds FCAR) ainsi que le Conseil de recherches en sciences humaines du Canada (CRSHC) pour l'aide financière reçue. Je remercie également les participants à la journée d'étude organisée sur le thème "Signification et portée des modèles d'évolution en économie et en sciences sociales », le 26 février 1997, à l'occasion du Séminaire d'épistémologie comparative du Département de philosophie de l'Université de Provence, de même que les participants au séminaire organisé le vendredi 11 décembre 1998 par le Groupe de recherche en épistémologie comparée, dont les remarques, questions et critiques ont été fort stimulantes. Je remercie tout particulièrement Alain Albert, Jean-François Bacot, Richard Collette, Stéphane D'Amour, Gilles Dostaler, Paul Dumouchel, Maurice Lagueux et Robert Leonard pour leurs suggestions qui m'ont aidé à réviser mon texte. 
reinforcing each other in the globally expanding market process. I finally try to show that Hayek's evolutionary perspective is crucially linked to his concern for population growth - in the precise sense of Malthus, which, as it is noted, profoundly influenced Darwin. But I also try to show that Hayek's evolutionary economics points nevertheless to good arguments against the "principle of population" (Malthus, 1798).

Le tont dernier ouvrage publié par Friedrich Hayck en 1988', soit quatre ans avant sa mort, met bien en évidence ce qui, sans doute, aura constitué le leitmotiv de toute son aeuvre. En effet, dans cet ultime essai, celui qui se présente lui-même comme le dernier économiste de l'école autrichienne dénonce une fois de plus a les erreurs du socialisme „, thème récurrent par excellence chéz ce prix Nobel d'économie (1974).

Si la dimension politique et idéologique de ce thème est obvie, elle n'est sans doute pas la seule, ni même peut-être la plus importante : la dimension scientifique de la question soulevée est certainement tout aussi importante et cruciale. La dimension épistémologique de ce thème ne saurait, non plus, échapper au regard averti. Car la pensée de Hayek est singulièrement traversée de part en part par une préoccupation afférente, à savoir la question des limites inhérentes au projet d'élaborer une science de la réalité économique. Dans la partie plus proprement philosophique de son œuvre, Hayek est en quelque sorte constamment obsédé par la nécessité de fixer des limites méthodologiques à la science économique, aussi bien comme science théorique que comme science empirique.

Mais si Hayek a été prompt à mettre au jour les a abus de la raison" en matière d'économique ${ }^{2}$, ce n'est que dans ses derniers écrits qu'il a pleinement dégagé et articulé la perspective théorique qui lui paraît la seule vraiment apte à permettre à l'économique de donner son plein rendement comme explication typique de la réalité,

1. F. A. Hayek, The Fatal Conceit: The Errors of Socialism. The Collected Works of F. A. Hayek, $3^{\mathbf{e}}$ éd., W. W. Bartley, Chicago, The University of Chicago Press, 1988, vol. I. Traduit en français sous le titre La présomption fatale. Les erreurs du socialisme par R. Audouin et révisé par Guy Millière, Paris, PUF (coll. "Libre échange »), 1993. Je citerai dorénavant ce texte en utilisant le sigle $F C$ suivi du numéro de la page, et je me référerai au texte de l'édition originale que je traduirai moi-même.

2. Voir F. A. Hayek, The Uses and Abuses of Reason: The Counter-Revolution of Science, Chicago, The University of Chicago Press, 1952, dont seule la première partie a paru en français grâce à la traduction de Raymond Barre, sous le titre Scientisme et Sciences Sociales (Paris, Plon, 1956). 
et cette perspective est de part en part évolutionnaire. C'est précisément cette perspective, dans ce qu'elle a de spécifique et de radical, que j’aimerais mettre en lumière ici. Hayek me paraît, en effet, apporter en 1988 des éclaircissements significatifs sur les tenants et aboutissants de la perspective évolutionnaire qu'il fait sienne.

Du reste, l'idée d'évolution biologique a été rendue possible, suivant Hayek, par l'étude du développement historique d'entités culturelles comme les langues naturelles, les codes de lois, les systèmes de mours et, au premier chef, les institutions économiques comme les marchés et la monnaie. C'est, d'ailleurs, de l'économique que Charles Darwin a tiré les idées de base de sa théorie de l'évolution, comme le signale Hayek $(F C$, p. 24) qui prétend que c'est la lecture d'Adam Smith en 1838 qui a protondément influencé Darwin 4 .

Pour Hayek, le cadre fourni par la théorie de l'évolution est généralisable et tous les événements naturels et culturels ne peuvent être réellement compris que s'ils sont envisagés dans une perspective évolutionnaire - donc aussi bien les processus biologiques que les processus économiques. Cependant, il est capital de voir qu'une différence essentielle empêche à toutes fins utiles qu'un traitement réellement darwinien ou néodarwinien soit fait des entités culturelles et de leur évolution. Hayek condamne sans appel la sociobiologie de Wilson et de ses épigones, eux qui auraient le tort de croire que les entités culturelles font l'objet d'une transmission génétique, alors que, suivant Hayek, elles sont apprises et transmises par imitation $(F C$, p. 24). La théorie sociobiologique est, pour Hayek, aussi impropre et inadéquate que la théorie qui veut que le langage, la loi, la moralité, e'est-à-dire, à toutes fins utiles, toutes les institutions

3. Avant de systématiser et de radicaliser sa perspective évolutionnaire dans The Fatal Conceit, c'est d'abord dans Law, Legislation and Liberty: A New Statement of the Liberal Principles of Justice and Political Economy (Londres/Chicago, Routledge \& Kegan Paul/The University of Chicago Press, vol. 1 :Rules and ()rder, 1973; vol. 2 : The Mirage of Social Justice, 1976 ; vol. 3 : The Political Order of a Free People, 1979) que Hayek a le plus développé ses idées à ce sujet, et tout particulièrement dans le premier volume. Cel ouvrage a été trarluit en français sous le titre Droit. législation et liberié, une nouvelle formulation des principes libéraux de justice et d'economie politique par Raoul Audoin, (Paris, PUF, vol. 1 : Règles et ordre, 1980; vol. 2 : Le Mirage de la justice sociale, 1982 ; vol. 3 : L'ordre politique diun penple libre, 1983).

4. Suivant Hayek ( $F C$, p. 24, note 1), il est attesté que Darwin a Ju en 1838 les Essa)s on Philosophical Subjects d'Adam Smith et qu'en 1839, il a lu The Theory of Moral Sentiments. Rien ne permettrait de croire, cependant, que Darwin aurait lu The Wealth of Nations. Mais il y a sans doute lieu d'insister également sur l'influence cruciale que joua Malthus sur Darwin, une dette que Darwin reconnaît pleinement dans son autobiographie (remarque communiquée à l'A. par J.F. Bacot dans une correspondance privée). 
sociales, soient des inventions délibérées de l'homme. Malgré cela, la perspective évolutionnaire reste absolument irremplaçable aux yeux de Hayek, car elle seule permet de mettre en évidence a l'évolution spontanée des règles de conduite qui président à la formation de structures auto-organisationnelles $₫(F C, p .37)$. Celte thèse mérite d'être examinée tròs sérieusement si l'on veut en découvirir le contenu et la portée véritables, et c'est à cet examen que je procéderai ici.

Mais avant de me lancer dans celte opération, une précation oratoire s'impose. On ne saurait dissocier daus l'ouvre de llayek ee qui relèverait, d'un côté, de la pure controverse idéologico-politique ou du débat philosophique et ce qui concernerait, de l'autre, avant tout, sinon exclusivement, la théorie économique, politique et juridique, à savoir la science sociale. C'est en tenant comple du haut degré d'intrication de ces préoceupations solidaires que je ferai mon analyse. Car cette situation n'est peut-ĉtre nulle part mieux mise en évidence que dans les écrits de Hayek, au demeurant nombreux, portant sur la constitution de ce qu'il appelle l'ordre spontané et sur la dynamique de ce qu'il estime constituer l'évolution culturelle de l'humanité.

\section{l'ordre économique spontané}

L'évolutionnisme hayékien prend appui sur un concept qui, à bien y regarder, sert de matrice à un ensemble multidisciplinaire de. théorisations, à savoir le concept d'ordre spontané $e^{6}$, et c'est pourquoi il convient d'abord de le présenter schématiquement et de faire voir la fonction que joue ce concept dans la théorie économique de Hayek?

5. Hayek écrit : « Le darwinisme social fait fausse route à plusieurs égards, mais l'intense mépris qu'il suscite aujourd'hui est aussi particllement dû au fait qu'il entre en contradiction avec la présomption fatale suivant laquelle l'homme peut faire en sorte que le monde qui l'entoure se conforme à ses souhaits. " $(F C$, p. 27)

6. Le concept d'ordre spontané appartient à ce qu'il est convenu d'appeler le "paradigme de l'auto-organisation" (voir L'Aluo-organisation: de la pliysique an politique, sous la dir. de P. Dumouchel et J.P. Dupuy, Paris, Editions du Seuil, 1983) el, sous une forme ou sous unc autre, on peut soulenir qu'il joue un rôle dans un grand nombre de théories appartenant aux disciplines les plus diverses comme, par exemple, la tectologie de A. A. Bogdanov, l'atrogenèse dle J. Smuth, le déterminisme stratifié de P. A. Weiss, la philosophie du processus de A. N. Whitehead, l'autopoièse de F. Varela et H. Maturana, les modèles cybernétiques de seconde génération de $H$. von Foerster, la thermodynamique des systèmes ouverts et des structures dissipatives de I. Prigogine, la théorie du chaos de B. Mandelbrot et la théorie des catastrophes de R. Thom. Philippe Nemo fournit une excellente analyse systématique de ce paradigme dans sa version hayékienne. Voir P. Nemo, La société de droit selon F. A. Hayek, Paris, PUF, (coli. "Libre échange v), 1988, p. 67-105.

7. J'expose sommairement dans cette section une partie de l'argument que j'ai articulé en détail dans un article récent consacré à explorer les tenants et aboutissants du concept d'ordre spontané. Voir "Spontaneous Order" dans John B. Davis, D. Wade Hands et Uskali Mäki (dir.), Handbook of Economic Methodology, Cheltenham, UK-Northampton, Edward Elgar, 1998, p. 477-484. 
Pour pouvoir rendre la conception hayékienne de l'ordre spontané intelligible, il importe d'abord de reprendre la distinction ontologique faite par lui entre cosmos et taxis. Le premier terme réfère non seulement aux systèmes naturels issus de l'évolution par sélection naturelle, par exemple les espèces de la biosphère, mais également aux systèmes sociaux qui sont apparus spontanément comme résultats de l'évolution historique de l'humanité depuis les temps immémoriaux. Le second terme est réservé par lui pour désigner exclusivement les systèmes qui sont issus d'un plan délibéré. Seuls ces derniers peuvent, suivant Hayek, être légitimement considérés comme " artificiels " puisqu'ils ont été fabriqués et sont, en ce sens, issus du génie humain. Il en va tout autrement des systèmes sociaux qui constituent des systèmes de règles de conduite et de perception et qui, tout en servant clairement aux individus à coordonner leurs plans d'action, ne sauraient être vus comme des constructions planifiées et délibérées par l'homme ${ }^{8}$.

Opposant pour cette raison, d'un côté, les institutions sociales ayant émergé spontanément au cours de l'histoire grâce à l'interaction des hommes (p. ex., les langues naturelles, les monnaies, les lois) et, de l'autre, les organisations mises en place progressivement par la libre volonté d'association et de coopération des agents individuels, Hayek définit l'ordre social spontané comme celui qui, tout en résultant pleinement de l'action humaine, ne résulte pas pour autant d'un dessein qu'un individu agissant seul ou que plusieurs individus agissant collectivement auraient pu entretenir et mener à terme $^{9}$. Hayek renoue ainsi avec la tradition britannique de la Common Law, c'est-à-dire avec des penseurs comme Bernard Mandeville, Adam Ferguson, David Hume, Josiah Tucker, Dugald Stewart, Thomas Reid et Adam Smith ${ }^{10}$. Si cette idée est pour la première fois exposée en détail, mais sous le mode métaphorique, dans la fameuse Fable des abeilles de Bernard Mandeville ${ }^{11}$, c'est

8. Voir F. A. Hayek, "Kinds of Order in Society ", New Individualist Review, vol. 3, $\mathrm{n}^{\circ}$ 2, 1963, p. 3-12 ; réédité dans K. S. Templeton Jr. (dir.), The Politicization of Society, Indianapolis, Liberty Press, 1979. Voir aussi F. A. Hayck, Law. Legislation and Liberty, vol. 1, chap. IV.

9. Voir F. A. Hayek, "The Results of Human Action but not of Human Design ", Situdies in Philosophy, Politics and Economics, Londres/Chicago, Routledge \& Kegan Paul/Lniversity of Chicago Press, 1967, chap. VI, p. 96-105. Traduit en français sous le titre "Résultats de l'action des hommes mais non de leurs desseins " dans Les Fondements philosophiques des systèmes économiques, textes de Jacques Rueff et essais rédigés en son honneur, Paris, 1967, p. 98-106.

10. Ferguson est d'ailleur's l'auteur d'une fornule-choc dont Hayek s'est très clairement inspiré : " Nations stumble upon establishments, which are indeed the result of human action, but not the execution of any human design. ` (A. Ferguson, An Essay on Civil Society, Edinbourg, Edinburgh University Press, 1767/1966, p. 122)

11. Bernard Mandeville, The Fable of the Bees, rééditée avec une introd. de F. Kaye, Londres, Oxford University Press, 1924 (1 ${ }^{\text {re }}$ éd. : 1723); nouvelle éd. avec une introd. de P. Harth, Harmondsworth, Pelican, 1970. Sur l'importance que Hayek accorde à Mandeville, voir son article initialement publié en 1967, « Dr Bemard 
assurément Adam Smith qui, le premier, l'a systématiquement utilisée en théorie économique. Pour Smith, en effet, la société constitue globalement une structure ou un système spontanément engendré, auto-organisé ou autorégulé el évolutil. Le problème crucial est alors de rendre compte du maintien de cette structure dans le temps. Ce qui assure la durabilité de la cohésion sociale dans le temps, e'est, pour Smith, la dynamique de l'interaction humaine elle-même, à savoir le fait que, bien que chacun cherche avant tout à salisfaire ses intérêts propres, les actions qu'on est ainsi conduit à poser soient profitables à tous : la rhapsodic humaine, pourrait-on dire, certes une partition musicale d'une infinie complexité, est jouée comme si une main invisible dirigeait l'orchestre.

Suivant Smith, toutes les actions individuelles sont en quelque sorte interdépendantes, si indirecte que soient éventuellement leurs connexions. Les individus agissent en s'adaptant autant que faire se peut à la situation qui est la leur. Pour ce qui concerne leur bien-être économique, ils se fient, suivant Smith, à l'information que les prix leur fournissent. Its cherchent ainsi à obtenir que leurs actions soient les plus profitables possible mais, évidemment, à moindre coût et en fournissant le moindre effort. Chacun trouve sa façon d'être et cherche à exploiter la situation à son propre avantage, quitte à spécialiser son champ d'activité. Du concert de la multitude des actions émerge un ordre économique que nul n'a en fait jamais vraiment voulu, que nul de toute manière n'était à même de faire advenir, mais un ordre foncièrement contingent et aléatoire où chacun trouve finalement la place qui, inexorablement, lui revient.

Une conséquence semble s'imposer d'elle-même à la suite de ces considérations : si les choses sont comme Mandeville et Smith prétendent qu'elles sont, alors nous sommes contraints de reconnaître avec eux la prééminence de l'économic de marché sur l'économie consciemment planifié $\mathrm{e}^{\mathrm{t} 2}$. Et il ne l'ait aucun doute aux yeux de Hayek qu'une authentique économie de marché, paree qu'elle est basée sur le système des prix, lui-même issu de la libre interaction des agents économiques, peut permettre une coordination des plans d'action individuelle qu'une planification économique centrale ne saurait produire.

C'est que la matière première d'une économie de marché est, selon Hayek, la connaissance - cette connaissance que seuls peuvent détenir des individus rationnels placés dans des situations

Mandeville "et repris dans ses New Studies in Philosophy, Politics, Economics and the History of Ideas, p. 249.266.

12. John Gray préfère considérer que cette thèse n'est pas partie intégrante de la théorie hayékienne de l'ordre spontané ; voir J. Gray, Hayek on Liberty, Oxford, Basil Blackwell, 1984 (2 éd. : 1986), chap. III : "The Idea of a Spontaneous Social Order ", p. 27-55 et chap. VI, section 2: "The Status and content of the idea of spontaneous order in society ", p. 118-125. En ce qui me concerne, c'en est le prolongement obligé, et c'est même la conclusion ouvertement recherchée par Hayek. 
absolument singulières et qu'ils sont les seuls à pouvoir comprendre tout à fait ${ }^{13}$. Car cette connaissance a trait à leurs projets, à leurs plans, à leurs contraintes particulières, à leurs préférences propres et à leurs objectifs personnels, tous éléments constituant autant de dimensions de la situation proprement incomparable à laquelle ils se trouvent individuellement confrontés.

Pour autant qu'il serait possible de totaliser l'information qui circule dans une société le moindrement développée, on en obtiendrait très vite une quantité si énorme qu'il devient impensable qu'un seul et même cerveau puisse l'emmagasiner. Les " données " seraient trop nombreuses pour être transcrites sous forme d'équations; mais à supposer qu'on puisse imaginer y parvenir, l'algèbre qưil faudrait construire serait si énorme qu'un tel calcul dépasserait l'entendement. Mais, de toute manière, cette information n'est pas totalisable, car elle est inévitablement dispersée entre d'innombrables agents individuels, qui n'en possèdent qu'une petite portion, et elle est condamnée à rester telle; qui plus est, chacun n'en dispose pas d'avance et ne l'obtient que lors de son intervention personnelle dans le jeu économique du marché, car cette information est strictement fonction de ce que pensent, de ce que désirent et de ce que préfèrent les autres, et ils ne le révèlent que dans ce qu'ils décident de faire. Le prix que quelqu'un est prêt à payer pour une marchandise - et l'on peut supposer que, dans une économie de marché, pratiquement tout a ou peut avoir un prix - n'est pas la propriété intrinsèque d'un bien ou d'un service : ce prix reflète essentiellement la valeur de ce à quoi cette personne est prête à renoncer pour obtenir le bien qu'clle convoite ou le service qu'elle cherche à obtenir des autres.

Il devient clair que, de ce point de vue, plus une économie pourra mettre à profit les connaissances des individus qui y sont actifs et plus elle sera efficace. Plus elle sera efficace et plus les individus y trouveront leur dû. Et c'est pourquoi il faut forcément reconnaître, insiste Hayek, qu'aucune économie ne samait surpasser l'efficacité de l'économie de marché puisque, ici, chaque individu est en principe laissé libre de ses entreprises, c'est-à-dire libre d'uliliser comme bon lui semble les connaissances qu'il acquiert, el puisque toute action individuelle a la possibilité de contribuer à acerôitue à terme le bienêtre des autres. II suffit done qu'un tel régime économique soit mis à l'abri de toute déviance et de toute délaillance par la mise en place d'un cadre juridique approprié pour que les individus y trouvent leur compte. Ei c'est parce que les individus sont tous susceptibles d'y trouver éventuellement leur compte que l'économie de marché

13. Sur cette question, qui occupe une place cruciale chez Hayek, voir en particulier ses deux articles: "Economics and Knowledge ", Economica, n. s., vol. 4, $n^{\circ} 13$, 1937, p. 33-54 (repris dans Individualism and Econonic Order, p. 33-56) et "The Use of Knowledge in Society ", American Economic Review, vol. 35, n 4, 1945, p. 519. 530 (repris dans le même ouvrage, p. 77-91). 
fournit spontanément, pour Hayek, un ordre social qui, sans être optimal absolument (ce qui n'a pas de sens de toute façon), constitue le meilleur ordre possible, tout bien considéré. Tel est l'usage que fait Hayek du concept d'ordre spontané.

\section{L'économie de marché comme ordre extensif}

Mais si important que soit le concept d'ordre spontané, il ne suffit pas à asseoir la perspective évolutionnaire de llayek. Loodre économique et social dont il faut rendre compte niest pas que spontané, il est également extensif (Hayek parle cn ce sens d'extended order). Cet ordre tend, en effet, à s'amplifier et à s'aceroître, et l'on peut, du reste, en observer l'expansion historique. Une telle analyse macroscopique est menée à bien par Hayek, quoique de manière. beaucoup plus expéditive qu'initialement prévu par lui, dans The Fatal Conceil ${ }^{14}$. C'est, du reste, dans cet ouvrage que le lien entre la perspective évolutionnaire et la critique du socialisme est le plus évident. Pour l'essentiel, l'argumentation de Hayek peut être rendue. de la manière suivante.

L'erreur principale de la théorie socialiste, qui croit possible de substituer un ordre économique supérieur, parce que consciemment planifié, à l'ordre spontané et extensif de l'économie de marché, est d'ignorer à toutes fins utiles la façon dont nous acquérons les connaissances qui nous sont économiquement et socialement utiles. Un tel apprentissage exige autre chose que la simple observation et l'expérience immédiate : il exige l'existence d'une tradition de laquelle nous puissions tirer inconsciemment des enseignements profitables. Pour ce qui a trait à l'existence économique et sociale des hommes, la tradition requise est d'ordre moral, au sens sociologique du terme : elle concerne les règles de la conduite quotidienne des affaires humaines.

Or, ce qui rend historiquement possibles le développement el la croissance économiques, e'est ultimement un ensemble cohésif de croyances morales qui, en elles-mêmes, sont rationnellement injustifiées, voire probablement injustifiables. Peut-ẹtre ne sont-elles

14. À l'origine, c'est un énorme ouvrage en trois volumes que Hayek voulait consacrer à la critique de ce qu'il appelle "la présomption fatale ". L'ouvrage auquel pensait Hayek alor's qu'il abordait ses quatre-vingts ans s'insèrait, en effet, dans un projet vaste et ambitieux qui vit le jour en 1978, celui de réunir à Paris, dans un immense débat international, d'un côté, les principaux théoriciens du socialisme et de l'autre, les principaux avocats de l'économie de marché (voir à ce sujet la préface éditoriale de William Bartley à l'ouvrage de Hayek, $p . x$ ). L'événement n'eut évidemment jamais lieu. Mais, comme l'explique Bartley, c'est pour des " raisons pratiques " que, finalement, ce débat n'eut pas lieu. "Comment. par exemple, seraient choisis les représentants du socialisme? Les socialistes eux-mêmes pourraient-ils seulement se mettre d'accord sur leurs représentants ? Et même en supposant qu'ils parviendraient à s'entendre, peut-on croire qu'ils auraient accepté de reconnaître l'issue d'un tel débat? Confesser publiquement ses erreurs n'est jamais chose facile à faire. "(ibid., p. $x$ ) 
pas même complètement détectables, tellement elles sont enfouies profondément dans la conscience des individus. Quoi qu'il en soit, la transmission intergénérationnelle de ces règles morales a lieu tout à fait à l'insu des individus : c'est inconsciemment que ceux-ci adoptent la plupart des normes de comportement qu'ils suivent pourtant scrupuleusement. Ces règles, pour Hayek, ont trait aussi bien à la perception qu'à la conduite et elles n'ont à vrai dire qu'une seule propriété vraiment déterminante sur le plan économique : elles favorisent le maintien et la croissance des groupes qui les suivent au détriment des groupes qui s'en écartent. C'est sur cet argument fondamental que Hayck assoit en fin de compte sa théorie de l'évolution culturelle. Celte théorie se fonde, en effet, sur l'hypothèse de l'existence d'une sélection des grompes humains du point de vue des performances économiques des individus qui en font partie ${ }^{15}$.

Il importe de voir que ces règles ne sont pas adoptées argumentativement ou délibérativement, au terme d'une enquête empirique ou d'un débat philosophique, comme si elles étaient empiriquement induites ou rationnellement inférées. C'est dire, bien sûr, que leur valeur adaptative n'est pas nécessairement reconnue consciemment. Ces règles morales permettent néanmoins, lorsqu'elles sont suivies, à des individus extrêmement distants les uns des autres, et appartenant à des groupes concurrents, de s'échanger de l'information par le système des prix et, ainsi, de coordonner leurs actions propres. Ce processus de coordination réciproque est, suivant Hayek, tout entier analysable en termes évolutionnaires d'adaptation à l'inconnu et il ne peut exister aucun substitut planifiable à ce processus d'ajustement automatique. En ce sens, une des tâches les plus fondamentales de la théorie économique est, pour Hayek, à la fois de montrer comment ce processus fonctionne el de dévoiler les limites qu'il impose aux hommes qui en dépendent entièrement pou leur' survie.

Toute conduite individuelle basce sur de telles règles morales peut être considérée comme un processus de maximisation sous contraintes : chaque individu appartenant à un groupe social prend part à de multiples échanges et fait usage d'une information foncièrement incertainc èt incomplète avec le dessein de s'assurer le. meilleur sort possible. Même si on peut modéliser de telles conduites sous la forme d'un jeu au sens de la théorie des jeux, ce processus ne peut jamais êlro assimilé, aux yeux de Hayek du moins, à un ealcul conscient et délibératif qui serait le fait d'un esprit individuel, et encore moins d'un "esprit collectif" ou d'un organe décisionnel

15. Dans The Fatal Conceit, Hayek croit utile et légitime d'appuyer ses conceptions personnelles sur la théorie de Wynne-Edwards (V. C. Wynne-Edwards, Amimal Dispersion in Relation to Social Behaviour, Edinbourg, Oliver \& Boyd, 1962), qu'on a beaucoup critiquée par la suite et que, sans doute, plus personne aujourd'hui ne soutient. Mais il serait inapproprié et incorrect de lier le sort des idées de Hayek sur la sélection économique des groupes humains à celles de Wynne-Edwards. 
unique et central. Dans le cadre de la théorie des jeux, une action se définit par ou se réduit à l'ensemble des conséquences qu'elle produit. Or, les conséquences des décisions innombrables prises par les agents individuels sont, pour la plupart du moins, à proprement parler incalculables et inconnaissables à l'avance : elles sont inanticipables parce qu'elles sont en majeure partie inintentionnelles. C'est, en conséquence, un tout autre point de vue qu'adopte Hayek sur le comportement économique et sur l'action humaine.

Suivant Hayek, la multiplication des actions individuelles donne lieu à la mise en place d'un ordre complexe qui non seulement s'autogénère spontanément, mais qui, de plus, a foncièrement tendance à s'étendre indéfiniment: cet ordre est par lui-même en expansion constante, saul' si, bien sûr, on y met un frein. L'ordre en question s'étend d'autant plus que les agents sont laissés libres de leurs décisions personnelles. II crồt à proportion que les individus qui se coordonnent entre eux sont non seulement de plus en plus nombreux, mais de plus en plus différents les uns des autres et qu'ils peuvent jouir de leur diversité grandissante au sein de structures de coopération de plus en plus élaborées.

Mais cet ordre économique, contrairement à ce que ses détracteurs lui ont souvent fait dire, n'est pour Hayek ni parfait, ni optimal, ni assuré, ni achevé, ce qui ne veut pas dire cependant que, par comparaison avec d'autres ordres possibles, il ne soit pas le plus efficace. Pour Hayek, les principales insuffisances ou déficiences de cet ordre expansif lui viennent habituellement de nos tentatives pour l'améliorer ou, pire, de nos actions pour l'empêcher de jouer son rôle qui, ultimement, en est un d'élimination du moins apte. De ces arguments, Hayek tire la conclusion que la théorie qui propose de remplacer l'ordre extensif de l'économie marchande (que Hayek appelle préférablement la catallaxie) par un ordre planifié commet une erreur d'ordre gnoséologique, car elle se trompe sur ce qui est réellement connaissable par l'agent économique individuel. Mais également, et plus gravement encore, cette théorie est déficiente sur le plan méthodologique, car elle n'envisage pas l'économique dans une perspective résolument évolutionnaire. Ces deux erreurs sont capitales aux yeux de Hayek : car les commettre conduit généralement à adopter une perspective illégitime sur la tâche de la théorie économique, dont on attend à tort non pas simplement qu'elle explique et permette de comprendre les principes fondamentaux du fonctionnement de l'économie, mais plutôt qu'elle outille l'économiste de manière à en faire un ingénieur planificateur qui pourra reconstruire la société sur des bases jugées plus rationnelles, et donc plus saines. Dans les sections qui suivent, j'examinerai plus en détail les arguments qu'avance Hayek pour justifier son point de vue. 


\section{Compétence économique et compétence épistémique}

Hayek reprend à son compte deux idées de Michael Polanyi ${ }^{16}$. D'abord, il convient d'admettre qu'il existe des types d'ordre mieux organisés et plus robustes que d'autres, voire, pour ce qui concerne les ordres proprement économiques, des types d'ordre plus efficaces que d'autres, notamment du point de vue de l'allocation des ressources en fonction des besoins exprimés. Pour étayer cette thèse, Hayek reprend de Polanyi l'idée que les types d'ordre qui s'avèrent les plus dynamiques ne sont pas monocentriques, mais plutôt polycentriques : ils sont donc très fortement décentralisés, pratiquement locaux, strictement fonction de paramètres qu'on ne saurait prévoir longtemps à l'avance, et apportent aux problèmes de la conjoncture des solutions imprévisibles et incertaines, temporaires et non généralisables.

La nature des problèmes économiques qui se posent dans les sociétés dont le développement est basé sur le commerce et l'industrie fait que les agents susceptibles d'apporter des solutions à ces problèmes sont les agents mêmes qui sont pris dans la situation où surgissent les problèmes. Ceux-ci comprennent le plus souvent la situation dans laquelle ils se trouvent sans se la représenter sous la forme d'un modèle théorique. En effet, le savoir mis en cunre par ces agents n'est pas du genre propositionnel ou théorique : il est du type de ce que Polanyi appelle le tacit knowledge, une connaissance non formulée, un savoir pratique portant davantage sur le comment que sur le pourquoi des choses. Loin d'être livresque, ce savoir est personnel : il est un savoir-faire que l'individu acquiert lorsque mis en situation pour décider de son sort et il ne se transmet que localement, c'est-à-dire aux individus placés à leur tour dans une situation identique ou analogue et qui sont prêts à imiter les solutions éprouvées par d'autres.

La connaissance tacite dont il est question ici se ramène à des habiletés personnelles acquises avec le temps, à la maîtrise de pratiques régies par des règles de perception el de conduite strictes que lindividu qui les suit ne serait pas capable de décrire explicitement et complètement. Ces règles sont profondément inconseientes et elles sont dautant micux maîtrisées que l'individu peut les suivre sans même y penses. Peut-être même y penserait-il 'qu'il ne pourrait pas expliquer pourquoi il les suit, si ce n'est que, manifestement, l'observance de ces règles donne bien souvent d'heureux résultats.

Ces multiples savoirs pratiques et ces innombrables compétences personnelles qui font fonctionner l'économie, nul n'aurait pu prédire

16. Voir Michael Polanyi, The Logic of Liberty: Reflections and Rejoinders, Chicago, The University of Chicago Press, 1951, mais aussi " The Determinants of Social Action * dans Erich Streissler et al. (dir.), Roads to Freedom : Essags in Honour of Friedrich A. von Hayek, Londres, Routledge \& Kegan Paul, 1969, p. 145-179. 
qu'on les trouverait utiles; nul ne peut savoir d'avance non plus combien de temps elles seront vues comme utiles; et, enfin, nul ne pent anticiper par quelles autres pratiques elles seront éventuellement remplacées à terme ${ }^{17}$. Ce savoir n'est pas de cenx qui s'emmagasinent et qui se tolalisent en un même cerveau : il est essentiellement fragmenté el distribué, tout comme il est de nature statégique et, pour une large part, de nature non réflexive, ce qui implique qu’il ne puisse s'acquérir que dans l'action. Cola explique que la connaissance qui permet à l'ensemble social de s'acliver et de se développer est foncièrement d'ordre individucl et personnel. Fi c'est parce qu'il en est ainsi qu'une coconomie contralement planifiée est toujours condamnée à moins bien fonchionner qu'une économie de marché basée sur la libre inceraction des producteurs (ou des entrepreneurs) et des consommateurs.

Cela explique enfin que la nature des informations écomomiques qui constitue ce type de comnaissance est mon totalisable dans un organe central de décision ${ }^{18}$. Car non seulement la tâche de recueillir et de mettre en ordre ces informations est infinie, mais, à supposer qu'on veuille et qu'on puisse les rassembler, il faudrait tollement de: temps pour les obtenir quelles seraient finalement inutiles au décideur, qui interviendrait irrémédiablement trop tard. En effet, ces informations privilégiées et nécessaires naissent du marché luimême, c'est-à-dire des multiples échanges incessants des agents individuels entre eux; qui plus est, les connaissances accumulées par les individus dans leur situation propre ne seraient d'aucune utilité, quand elles ne seraient pas tout simplement inaccessibles, à ceux qui ne se trouveraient pas exactement dans la même situation qu'eux. L'idée d'une économie, voire d'une société, centralement planifiée et. contrôlée s'avère donc une chimère.

Le fait que l'ordre économique efficace soit spontané al qüil se fonde sur la mise en réseau épistémique des agents économiques

17. Cet argument rejoint manifestement l'argument erucial de Popper que l'on trouve clans la préface à Misère de l'historicisme (Paris, Ploni, 1956) et faisant valoir que l'on ne peut évidemment savoir l'avance à proprenent parler quelles comnaissances on possédera demain.

18. Hayek soutient qu'il est absolument crucial de saisir la différence "between an order which is brought about by the direction of a central organ such as the brain, and the formation of an order determined by the regularity of the actions cowards each other of the elements of a structure ". II conclut ainsi sa remarquable analyse : "Finally, such spontaneous orders as those of societies, although they will often produce results similar to those shich could be produced by a brain, are thus organized on principles different from those which govern the relations between a brain and the organism which it directs. Although the brain may be organized on principles similar to those on which a society is organized, society is not a brain and must not be represented as a sort of super-brain, because in it the acting parts and those between which the relations determining the structure are established are the same, and the ordering task is not deputized to any part in which a model is preformed. " (F. A. Hayek, "Notes on the Evolution of Systems of Rules of Conduct [The Interplay between Rules of Individual Conduct and the Social Order of Actions] ", Studies in Philosophy, Politics and Economics, Chap. IV, p. 73, 74). 
individuels implique pour Hayek que la société ne puisse être conçue comme s'il s'agissait d'un hypercerveau supraindividuel et unique. Cette idée s'inscrit dans le prolongement direct des arguments développés par Hayek concernant l'importance de la connaissance dans le fonctionnement économique d'une société où les individus jouissent de la plus grande liberté d'action possible. Et ici encore, l'influence d'Adam Smith sur Hayek est tangible.

Adam Smith, en effet, a bien mis en évidence que l'homme aboutit à un résultat global qu'il n'a pas pu rechercher intentionnellement $^{19}$, a savoir, pour le dire bref, la mise en place de la société elle-même danss ses multiples institutions. Il ne faudrait donc pas croire que la société résulte d'un grandiose projet que les individus auraient collectivement concocté ou d'un contrat juridique qu'ils auraient consciemment passé entre eux. Mais il y a plus : si la société n'est pas issue de la volonté générale, elle ne fonctionne pas davantage comme si elle était contrôlée par un centre de décision global où toute l'information convergerait et d'où toute décision émanerait : la société n'est pas comme un individu qui pense, qui planifie et qui décide. Non seulement la société n'est pas représentable comme un hyperindividu, mais on ne saurait pas davantage penser qu'elle est un système qu'un centre de décision collectif, fonctionnant comme un supercerveau individuel, pourrait gérer à sa guise. Partant, on ne saurait concevoir la société comme si les rapports économiques entre individus pouvaient être coordonnés efficacement par un gouvernement central aux pouvoirs illimités, qui prétendrait pouvoir programmer arbitrairement le système de ces rapports, voire le reprogrammer à volonté et le diriger en fonction d'un plan d'ensemble.

\section{Coordination économique et compétence morale}

Pour réellement expliquer le fonctionnement de la coordination économique, il faut rendre compte des conditions de possibilité historiques de l'avènement de ce processus et de son renforcement progressif. Une hypothèse permet à Hayek d'expliquer globalement que les individus sagglomèrent en communautés af forment une société : ectte hypothése veut que les individus soient amenés à se regrouper sur la base des regles de conduite el de perception qu'ils suivent. Ainsi, Hayek est d'avis que, selon la tradition morale dans laquelle un indiviclu sera éduqué ou à lacpuelle, devenu adulte, il se ralliera, cet individu se retrouvera immanquablement dans une

19. Ainsi s'exprime Adam Smith : " Man is led to promote an end which was no part of his intention * (A. Smith, An Inquiry into the Nature and Causes of the Wealth of Nations, The Glasgow edition of the works and correspondance of Adam Smith, R. H. Campbell et A. S. Skinner [dir.], Oxford, Clarendon Press, 1776/1976, vol. 2, p. 456). Voir aussi A. Smith, The Theory of Moral Sentiments (The Glasgow edition of the works and correspondance of Adam Smith, D. D. Raphael et A. Macfie (dir. de publication), Oxford, Clarendon Press, 1759/1976, vol. 1, p. 58. 
société tout à fait distincte de toutes les autres et, partant, il prendra place dans une économie qui aura un fonctionnement tout à fait spécifique ef qui sera, en conséquence, plus ou moins performante, étant entendu que toutes ne sont pas cegales sous ce mapport.

Tout compte fait, même si Hayck nous parle de tuatition morale, d'histoire et d'anthropologie, le ressort de l'argument évolutionnaire quil déploie est foncièrement économique. Il se resume en fait à deux axiomes centraux de la théoric économique. D’abord, Ilayek insiste pour dire que seules les différences entre les prix anticipés pour divers biens et services et leur coût respectif, différences que fixe l'ordre autogénéré du marché, enseignent à l'individu comment il peut le mieux contribuer au pool économique el social, pool eluquel chacun reçoil toujours inévitablement, suivant Ilayek, à proportion de ce qu'il y contribue. Ensuite, llayck fait valoir fue seuls un calcul et une distribution opérés en termes de prix de marehé peuvent permettre à chacun d'user au mieux des ressomrees qüil y trouve, de: guider sa propre production de maniène à ce qu elle serve à satisfaire des fins situées bien au-delà de co qu"il lui est possible de percevoir comme producteur de biens et de services, donc de faire en sorte que l'individu participe utilement et de manière productive au processus économique de l'échange.

Dans une économie de marché développée, les individus font satisfaire leurs besoins personnels par l'action d'autres individus qui, pour la plupart, ne les connaissent pas et qu'ils ne connaissent pas davantage personnellement; toutes ces personnes agissent en se laissant guider seulement par les signaux du marché. Comme Hayek le fait remarquer, la coordination des agents dans la catallaxie n'exige pas de savoir collectif. En un sens, Hayek nous oblige même à constater que non seulement cette exigence n'existe pas, mais que c'est plutôt le contraire qui est nécessaire. Iin effel, les pratiques économiques qui induisent l'ordre extcrusil, c'est-à-dire la coordination des agents multiples - et cola est d'autant plus varai que les individus dont il sagit appartiemnent à des groupes de plus en plus distants les uns des autres -.., peuvent, cortes, etre communes el partagées, mais elles doivent se baser sur la poursuite d'objectifs différents d'un individu à l'autre, sans quoi l'échange productif ne pourrait avoir lieu. Or, cette différence dans les fins poursuivies n'a elle-même de chance d'être efficace que si elle implique que chaque agent individuel ait ultimement accès à de l'information privilégiée sur laquelle il réglera sa conduite ( $F C$, p. 43).

L'interaction économique des individus est donc un processus que l'on pourrait qualifier d'autoréglé. Les règles de conduite auxquelles les individus se soumettent sont celles que leur culture traditionnelle propre leur a inculquées sous la forme d'une morale. Et c'est une tradition morale tout à fait spécifique qui se trouve à la base de l'économie de marché. Comment rendre compte autrement du fait qu'une multitude d'individus forment, le cas échéant, un 
ensemble au sein duquel ils développent leur rayon d'action et se donnent éventuellement un champ d'activité spécialisée ?' Comment expliquer que ceux-ci acceptent de coopérer avec d'autres, qu'ils jouent le jeu de l'échange réciproque des biens et services et s'assurent ainsi mutuellement de pouvoir réaliser les conditions économiques de leur bien-être? Hayek croit pouvoir précisément trouver la réponse à cette question dans ce qui caractérise le système des valeurs qui, dans la catallaxie, guident l'action des individus en fixant les règles de perception et de conduite qu'ils seront amenés à suivre de manière à s'intégrer au groupe économique qui garantit les conditions de leur survie $e^{201}$.

En faisant l'hypothèse de l'existence d'une évolution culturelle de l'humanité, llayek entend meltre au jour le mécanisme par lequel s'opère historiquement la sélection naturelle des traditions morales, l'idée étant de parvenir à expliquer de la sorte la prééminence de l'économie marchande sur toutes les autres formes de vie en société. L'hypothèse de l'évolution culturelle suppose, en effet, que les groupes humains soient en compétition les uns avec les autres. Ces divers groupes cherchent à survivre et à se développer, voire à se renforcer et donc à s'accroître. Ce à quoi Hayek se réfère lorsqu'il parle de a tradition morale », c'est au système sociologiquement observable des mœurs qu'il juge non seulement propices, mais à proprement parler indissociables du régime de vie catallactique, c'est-à-dire de la vie humaine en économie marchande. Ces mours sont effectivement réglementées, mais pas forcément par législation ; elles ne sont pas mềme explicites le plus souvent, et sont encore moins partagées comme une connaissance commune. Pour Hayek, elles ne sont pas même toujours bien comprises par ceux qui les suivent, eux qui y obéissent en fait aveuglément. Elles ne sont pas instinctives pour autant puisque, comme Hayek l'affirme, elles sont apprises. Elles sont, en effet, transmises de génération en génération par le jeu de l'éducation el, plus globalement, chacun les acquiert pour lui-même en participant à la même culture que les individus du groupe où il passe son existence.

Ces règles ne sont done pas choisies délibérément par quiconque, cest-à-dire au terme d'une réllexion évaluative, justificative et normative. Bien au contraire, elles simposent aux individus puisqu'elles contribuent à la sélection de ceux qui les suivent au détriment de ceux qui les ignorent et à la sélection de leur groupe d'appartenance au détriment d'autres groupes avec lesquels le leur compétitionne. Hayek soutient même que, lorsqu'elles sont thématisées pour elles-mêmes, ces règles morales sont la plupart du temps décriées, parce qu'elles sont perçues comme contredisant très souvent l'éthique rationalisée, qu'elle soit d'origine religieuse ou

20. Voir à ce propos a Competition as a Discovery Procedure ", New Studies in Philosophy, Politics, Economics and the History of Ideas, p. 179-190. 
philosophique. En l'occurrence, ces règles ne doivent en aucun cas être confondues avec les préceptes d'une éthique universelle à la manière de Kant. Suivant Hayek, dont la perspective, faut-il le rappeler, est strictement économique, seules les règles morales qui s'avèrent les plus profitables de ce point de vue s'imposent et perdurent au sein d'une tradition, car, comme Hayek le fait valoir à bon doit, "seule la règle la plus profitable nourrira le plus de monde du fail quelle sacrifie moins que ce qu'elle ajoute s (FC, p. 46).

II n'est pas hanal de considérer, comme Hayck le fail, que les moxurs sur lesquelles se fonde l'économie de marché ne soient ni instinctives ni rationnelles : elles se situent, soutient Hayek, "entre rinstinct et la raison ". Qu'est-ce à dire?' Les mours instinctives sont, pour Hayek, les moxurs d'origine, à savoir les mours primitives qui sont tout à la fois tribales, altruistes et consensuelles. Les pratiques qu'elles induisent sont celles de groupes distinets dont les membres visent à s'entraider de manière à pouvoir survivre individuellement. L'altruisme primitif assure la cohésion du groupe et, à celte époque reculée, la fusion dans le groupe constitue pour l'individu le meilleur moyen de durer ou de garantir les conditions de son existence. Loin qu'à l'origine, l'instinct de préservation de l'individu requière de lui qu'il cherche à satisfaire son intérêt personnel, son action est plutôt réglée par la volonté de préserver à tout prix son appartenance au groupe. Et, pour autant qu il y ait du sens à contraster globalement entre elles deux traditions morales, l'une altruiste et l'autre centrée sur l'intérêt personnel, Hayek est d'avis que la première a certainement vu le jour avant la seconde, mais que la seconde a progressivement pris le pas sur la première précisément à cause de l'avantage évolutif qu'elle se trouvait à procurer aux groupes qui en vinrent à l'adopter.

Ainsi, les règles de conduite auxquelles Hayek se réfère et qui définissent la compétence morale des individus ne sont pas partie intégrante de la raison si, par raison, l'on entend l'exercice réflexil' de la faculté de jugement et l'adoption délibérative d'une maxime de conduite que l'on sait soumise à quelque impératif calégorique. La morale qui sous-tend la catallaxie n'origine en rien de la raison raisonnante et de l'argumentation spéculative. Cotte morale est faite de règles purement abstraites ${ }^{21}$-- elles sont formelles en ce sens - et elles ne sont en rien des stipulations raisonnées ou des injonctions mûrement réfléchies. Elles n'ont qu'une fonction spécifique, soutient Hayek, et c'est de contraindre les moyens d'action des individus dans le but de fixer des limites aux fins qu'il leur sera loisible de poursuivre dans leurs activités. Ces règles sont, dès lors, foncièrement négatives puisqu'elles ne visent qu'à limiter le rayon d'action individuelle $(F C$, p. 49).

21. Voir à ce sujet "The Primacy of the Abstract " dans A. Koestler et J. R. Smythies (dir.), Beyond Reductionism, Londres, 1969 ; repris dans les New Studies in Philosophy, Politics, Economics and the History of Ideas, p. 35-49. 
En conséquence, c'est, aux yeux de Hayek, l'idée d'une morale consensuelle assurant le socle d'un contrat social qui constitue a la principale source de la présomption fatale du rationalisme intellectuel moderne qui promet de nous ramener à un paradis perdu où nos instincts naturels, plutôt que les restrictions que nous avons appris à leur appliquer, nous permettront de " subjuguer le monde", comme le livre de la Genèse nous instruit de le faire ( $F C$, p. 49). L'idée de substituer une éthique démontrée à l'éthique révélée, qui est, en particulier, le projet d'Auguste Comte ${ }^{22}$, dans la mesure où elle fait partie du projet extravagant de rationaliser le réel en le soumettant au plan de l'esprit humain, est une idée carrément absurde pour Hayek. Le rationalisme constructiviste, parce qu'il pense pouvoir faire mieux que la morale dont nous avons hérité (et la morale judéo-chrétienne n'est pas davantage assimilable à la morale catallactique dont parle Hayek), pose très exactement le problème de la supériorité alléguée ou supputée de ce qui est rationnellement construit sur ce qui est évolutivement acquis. Or, le point central de la doctrine hayékienne tient justement dans cette affirmation que nos moeurs, et donc nos institutions concernant la propriété, la liberté et la justice, ne sont nullement nos créations puisqu'elles sont en fait la résultante de l'évolution.

Il importe cependant de remarquer que, même si Hayek fait l'hypothèse d'une sélection des groupes, son scénario explicatif fait exclusivement intervenir des agents individuels et jamais les groupes auxquels ces agents appartiennent. Car l'ordre économique ne s'étend que parce que des individus tentent de pénétrer de nouveaux groupes et que, de part et d'autre, plusieurs individus peuvent $y$ trouver leur intérêt. Qui plus est, aussi bien vis-à-vis de son groupe d'appartenance propre que du point de vue du groupe où il vise à percer, l'agent individuel agissant comme un entrepreneur doit posséder une information qu'il est à peu près le seul à avoir ou, en tout cas, dont il est pratiquement le seul - ou le premier et donc le seul pour un temps - à pouvoir tirer parti $(F C$, p. 43). Une innovation ne peut en effet s'implanter dans un groupe que si l'individu qui s'en fait le promoteur possède sur tous ses concurrents un avantage comparatif appréciable. Hayek est d'avis, cependant, qu'une telle innovation doit également procurer aux membres du groupe où l'individu-entrepreneur introduit la nouveauté un avantage qui fait une différence significative pour le taux d'expansion de ce groupe puisque, autrement, elle n'aurait aucun impact économique. C'est là le seul sens de cette théorie de la sélection dite, à tort ou à raison, des groupes.

22. La remarquable analyse de Comte par Hayek se trouve aux sections 3 (p. 129-142) et 6 (p. 168-188) de la deuxième partie de The Counter-Revolution of Science, mais surtout dans la troisième partie, consacrée à Comte et Hegel, du même ouvrage (p. 189-206). 
Quoi qu'il en soit, plus l'ordre marchand s'étend et plus il devient diffieile à un individu donné de savoir exactement quels sont les besoins particuliers que son action lend à satisfaire et quels sont les individus qui profilcront du résullat de ses actions. Par ailleurs, le proeessus du marché est tel que nul ne réussit à y pereer et à s'y tailler une place šil ne sait que ce que tous savent déja ; mul ne connât de suceès personnel sil ne cherche à laire affaire quaves: reux qui ont exactement les mêmes besoins, les mêmes oljectifs et les mêmes comaissances que lui. L'économie de marché pour les groupes et l'évolution biologique pour les espèces ont done ceci de commun quion y connaît du suceès - reproduction d'un côté et expansion de lautre - que si l'on dispose d'un avantage comparatif sur ses concurrents.

Or, dans un rapport marchand, il n'est nul besoin de connaîtere lautre personnellement et d'être conmu de lui pour qu'un échange nous lic à lui et fonctionne correctement : il est tout à fail concevable: en ee sens que lintérêt public soit servi sans que les intervenants aient la moindre intention de laire autre chose que de s'intéresser à eux-mêmes et à ceux auxquels ils s"identifient. C"est ainsi que devient possible une structure auto-organisationnelle de grande envergure la catallaxie -- dont la mise en place comme structure d'ensemble n'est en rien redevable aux desseins de l'esprit humain. Loin qu'un savoir commun ait présidé à la destinée de cette structuration encore en plein essor aujourd'hui, c'est exclusivement la compétence épistémique de chacun, un savoir essentiellement individuel et privé, non communicable et personnel, qui peut permettre de rendre compte de l'émergence d'un tel ordré économique extensif. Cet ordre est effectivement en prise sur les actions de chaque individu, dont certaines réussissent sans que lindividu qui les commet ne puisse jamais savoir quelles en sont toutes les consciquences, of dont certaines échouent sans qu'il ait toujours pu anticiper les raisons de l'échec. Ce que l'économic de marehé rend possible, e'est que chacun use de son savoir propre à ses fins propres tout en ignorant presque tout de l'ordre dans lequel il doil insérer son action pour qu'elle porte des fruits $(F C$, p.47).

Tout autant que la compétence épistémique, la compétence morale des individus assure done leur coordination dans l'économie de marché. Si l'ordre résultant constitue une structure autoorganisationnelle ou spontanée, c'est qu'il s'agit d'un " macro-ordre " $(F C$, p. 37), d'un ordre évolutif et extensif. A ce titre, on ne peut soutenir que cet ordre ait une origine volontaire ni qu'il suive un dessein conscient. Dans la mesure où cet ordre est un processus de coordination dynamique et ouvert sur le futur, il n'a aucune linalité assignable. Cela veut dire qu'à défaut de laisser cet ordre se développer, se ramifier, s'imposer par lui-même partout où il ne s'est pas déjà infiltré (Hayek envisage même sans sourciller la possibilité du développement industriel de l'Antarctique), et à défaut de le 
laisser se complexifier partout où il a déjà fait son œuvre, l'ordre en question régressera, il diminuera d'intensité, il se disloquera et il dégénérera. Il aura tendance à céder la place non pas tant au désordre ou à l'absence d'ordre, mais plutôt au type d'ordre économique préexistant à l'économie de marché, entraînant du coup, insiste Hayek, non pas la « paupérisation du prolétariat „ (concept dont Hayek estime qu'il est absolument vide de sens) et pas même la mort des plus démunis, mais, plus radicalement, l'inexistence (au sens de la non-naissance) de millions d'êtres humains. Car l'analyse économique évolutionnairo est à même de révéler, suivant Hayek, que les règles de conduite qui ont émergé spontanément ont généralement tendu à favoriser la survie de l'espèce humaine ${ }^{23}$. C'est ici, dans une thèse qui se présentera comme anti-malthusienne, que l'évolutionnisme économique de Hayek prend tout son sens.

\section{Une optique populationnelle}

Il va de soi, pour Hayek, que les seules restrictions que puissent s'imposer les individus dans leur interaction économique sont celles qui limiteraient l'expansion de l'ordre qui les fait exister. Car cet ordre économique est ce qui leur permet d'avoir une progéniture et, ainsi, une descendance éventuelle. Cet argument démographique ou populationnel joue un rôle crucial chez Hayek, qui consacre de très brillantes analyses aux théories de Thomas Malthus dans son ouvrage de $1988^{24}$. Car l'ordre économique extensif est, par définition, l'ordre qui permet au plus grand nombre de se nourrir, de survivre et de se multiplier - argument évolutionniste par excellence.

Aux yeux de Hayek, il faut se débarrasser à tout prix de l'idée qu'il y aurait une évolution culturelle qui succéderait dans le temps à l'évolution naturelle et qui, partant, serait d'une autre nature. Il n'y a pas, pour Hayek, la biologie d'abord et la raison ensuite, car la raison est un acquis, et peut-être l'acquis fondamental, du processus biologique lui-même. La raison n'est pas le produit tardif, postérieur et done supérieur, de l'évolution : elle est tout entière un produit de l'évolution. L'idée d'une raison née de l'évolution et encore en train d'en émerger, dirigeant les opérations, est, pour Hayek, une contradiction dans les termes ${ }^{25}$.

23. Certes, comme y insiste Hayek, "le simple fait de reconnaître que (ces) règles tendent généralement à être sélectionnées, par l'intermédiaire de la eompétition, en fonclion de leur valeur pour la survie des hommes ne les soustrait pas à la nécessité d'un examen eritique " $(F C$, p. 20). Mais, pour Hayek, eet examen doit se faire du point de vue de la théorie économique et non pas du point de vue de l'éthique philosophique.

24. Voir le chapitre VIII de The Fatal Conceit : « The Extended Order and Population Growth •, p. 120-134.

25. Hayek se fait cinglant à ce propos et il écrit : "L'idée que la raison, elle-même créée au fil de l'évolution, serait maintenant en mesure de déterminer sa propre évolution future $[\ldots]$ est en elle-même contradictoire et peut être aisément réfutée $[\ldots]$. En tout état de cause, l'idée qu'à un certain moment donné du temps un 
Mais alors, parce que la raison humaine n'est elle-même que le produit de l'évolution, elle ne saurait mettre en branle un processus qui transcenderait le processus qui l'a fait émerger dans le temps et qui lui serait supérieur. Ce n'est pas l'esprit qui a fait la civilisation el c'est encore moins l'esprit qui en a dirigé le cours, car esprit humain et civilisation sont des expressions qui font incexomablement référence à des réalités conjointes qui se sont développées concurremment et qui, à ce litre, sont les effets synchronisés d'une cause commune. Il ne saurait être question pour layek de penser que l'esprit humain a produit la civilisation, comme si le processus civilisationnel navait pas lui-même engendré la rationalité.

J'aimerais soutenir ici que ce qui fait que la perspective théorique de Hayek est systématiquement et radicalement évolutionnaire, c'est que Hayek adopte ce que je suis tenté d'appeler une optique populationnelle. Et peut-être est-ce dans ce qui définit en propre ce point de vue que sarticulent et se joignent le plus fortement chez Hayek, dans une argumentation qui entremêle le parti pris idéologique et les considérations théoriques tirées de la science. économique, la critique du socialisme et la défense du libémalisme comme philosophie économique et sociale.

Rappelons d'abord, pour le bénéfice de la discussion, que Marx reconnaît l'existence d'un mécanisme régulateur de distribution des ressources. Cependant, suivant Hayek, Mises fait bien ressortir que Marx pense avoir mis à découvert les déficiences irrémédiables de ce mécanisme en montrant qu'il est aveugle : les producteurs sont aux prises avec l'incertitude de l'action des autres, se retrouvant ainsi systématiquement dans des situations difficiles et déplorables. Dans la perspective socialiste, c'est le plan économique qui doit justement avoir pour fonction de remplacer la mécanique aveugle par une mécanique prévoyante : évitant les aléas du marché, le plan permettrait alors d'atteindre à une productivité plus grande en rendant possible une affectation plus rationnclle des ressourees.

Hayek avance l’idée que, plutôt qu’un mécanisme, le marehé est un processus systémique qui englobe tous les agents, un processus qui a tendance à s'étendre puisqu’il est en expansion contimue et qu il ne. permet à personne de s'en abstraire. Partant, ce processus n'est en rien une machine qui aurait été construite par l'homme, mais il n'est pas davantage un artefact que les économistes pourraient avoir le loisir d'examiner à leur guise en le démontant pour ensuite le remonter différemment. Rappelant comment s'est opérée la mise en place des pratiques commerçantes, Hayek met en évidence que certaines mœurs (hospitalité, protection, droit de passage, etc.) ont dû apparaître en même temps qu'elles, les deux se renforçant

dessein conscient entra en scène et prit le relais de l'évolution substitue ce qui ne saurait être qu'un postulat sumaturel à une authentique explication scientifique. " (FC, p. 22) 
mutuellement. Hayek ne peut également manquer de constater en particulier que le sédentarisme et l'urbanisation en furent, à terme, les conséquences inéluctables.

C'est cet accroissement de la densité de la population dans certaines localités qui provoqua progressivement des occasions de spécialisation et qui entraîna finalement la division du travail, ce qui causa l'augmentation de la densité de la population, entrainant à son tour l'augmentation du revenu per capita, puis, à nouveau, un accroissement de la population, et ainsi de suite. L'évolution socioéconomique suit un pattern iclentifiable ${ }^{26}$, et qui est en un sens assimilable à un mécanisme d'autorégulation de la densité de la population par la production de biens et services, et de la quantité de richesse par l'aceroissement démographique -.. une sorte de dialectique à deux temps oì le premier moment contrôle l'autre, et réciproquement.

On comprendra que Hayek n'hésite pas à s'en prendre ouvertement aux pratiques malthusiennes el à dénoncer ce qu’il considère être, en cette fin de millénaire, une crainte injustifiée de surpopulation planétaire ${ }^{27}$. C'est pourquoi on conviendra aisément que l'optique populationnelle ne saurait paraître légitime en théorie économique que si les arguments cruciaux de Malthus sont déboutés de leur prétention à la validité, et c'est la tâche que se donne Hayek. Mais même s'il entend prendre le contre-pied du point de vue de Malthus, Hayek est le premier à reconnaître l'importance de la contribution de Malthus à notre compréhension des rapports entre économie et population. Se fondant sur un modèle théorique d'économie à deux facteurs de production, Malthus, qui est également lun des découvreurs de la loi des rendements décroissants, ne pouvait qu'ête conduit à conclure que toute augmentation du nombre des travailleurs provoquerait une réduction de la produchivité marginale, el done du revenu des travailleurs, particulièrement dans la situation où les meilleures terres auraient été occupées par des groupes de taille optimale.

Hayek ne dénigre en fait ni l'un ni l'autre des deux théorèmes fondamentaux de: Malthus : il prétend seulement que, la situation ayant dramatiquement changé depuis la parution de l'Essai sur le principe de population (1798), la thérie maluhusienne ne sapplique tout simplement plus. En effel, pour Hayek, une fois que le facteur

26. Concemant la problématicque épistémologique de ce que Hayek appelle la pattern recogmition et la possibilité de la pattern prediction, voir son article "The Theory of Complex Phenomena ", dans Studies in Philosophy, Politics and Economics, chap. II, p. 22-42. Ce texte a été traduit en français par Alain Boyer sous le titre u La theorie des phénomènes complexes "(Cahiers du C.R.E.A., vol. 13, 1989, p. 245294 ; introd. d'A. Boyer : p. 247.254; texte de Hayek : p. 255.294).

27. "L'idée moderne que la croissance de la population risque d'entrainer la paupérisation à l'ampleur de la planète est tout simplement une erreur. Cette erreur est en grande partie due au fait que l'on simplifie à outrance la théorie malthusienne de la population. " ( FC, p. 121-122) 
travail cesse d'être une entité homogène, el c'est ce qui se produit effectivement sous la pression évolutive de la spécialisation et de la diversification, lintensification des échanges, combinée avec l'amélioration des techniques de communication et des moyens de transport, provoque immanquablement une densification progressive de la population. Dans un tel contexte, la division du uravail amène à son tour le développement de nouveaux facteurs de production, augmentant alors peu à peu la productivité. Toute nouvelle habileté apparaissant au sein de la population active devient un nouveau facteur de produclion mare el précieux : celle nouvelle habileté prend place dans un ensemble oì elle ne survit que si elle stavere: complémenlaire aux aulres déjà en place, à moins qu'elle ne parvienne à déloger les fackeurs concurents, mais de produclivité moindre. Comme y insiste Hayek, la spécialisation volonlaire est simplement guidée par l'anticipation de différences significatives dans le niveau de rétribution éventuel $(F C, p .122)$.

Mais alors, la conclusion semble s'imposer d'elle-même : loin de provoquer une paupérisation progressive, l'augmentation de la population dans une économie de marché en libre expansion lait que le travail peut atteindre tendanciellement des rendements croissants plutôt que décroissants. Puisqu'une population globale plus dense peut aussi en venir à se servir d'outils et de techniques de production de plus en plus efficaces, et puisque les échanges commerciaux permettent d'équilibrer les niveaux de production el les taux de productivité entre régions, il est clair que la théorie de Malthus ne sapplique plus dans une telle situation. Car non seulement laugmentation de la population provoque l'enrichissement de plus de personnes, mais, qui plus est, indirectement, cette augmentation démographique cause à terme -- et pour des périodes indéfinies, insiste Hayek - une intensification de la densité démographique globale $^{28}$. Ce que Malthus n'a pas vu, selon Hayek, c'est que l'augmentation pure et simple du nombre potentiel de producteurs ef de consommateurs amène avec elle à terme l’intensifucation de la diversification, et done qu'elle engendre de nouvelles possibilités de spécialisation, ce qui à son tour a exige une extension du réseau des services indirects réciproques que fournit le mécanisme des signaux du marché » $(F C, p .123)$. Considérée à long terme, une lelle évolution économique ne peut done qu'être globalement bénélique puisque de plus en plus de monde en tire avantage.

Le moment est venu de conclure cette analyse. Parti de l'idée que l'ordre économique et social est, suivant Hayek, spontanément engendré, le processus de l'économie de marché a ensuite été caractérisé comme un processus en pleine expansion, pour autant qu'il soit laissé à lui-même dans un cadre juridico-politique qui le

28. Hayek soutient avec raison que " $[\mathrm{Ce}]$ n'est pas le fait qu'il existe un plus grand nombre d'êtres humains, mais bien le fait qu'il y a um plus grand nombre d'êtres humains différents qui induit une croissance de la productivité * $(F C$, p. 122), 
favorise le plus possible. Cherchant à expliquer cette dynamique, Hayek met en évidence que c'est l'opération de règles de conduite et de perception conformes à des normes morales traditionnelles profondément inconscientes qui peut le mieux en rendre compte. De telles règles, toutes négatives qu'elles soient et si peu conformes soient-elles aux canons de l'éthique philosophique, procurent aux individus qui les suivent un avantage évolutif certain : les groupes dont ceux-ci font partie dominent l'économie.

Les groupes économiquement dominants tendent ainsi à crô̂tre et à déloger les groupes d'individus dont la culture n'obéit pas aux mêmes régles morales. $\hat{\Lambda}$ plus long terme, il est prévisible que l'évolution favorisera nettement ces groupes dont l'expansion ne peut être vue que comme inévitable et indéfinie. L'évolution culturelle est ainsi mue par l'extension de la catallaxie qui, pour Hayek, est tout à fait souhaitable, sinon inévitable. Car, en retour, la domination mondiale de l'économic de marché force la prédominance des moeurs qui la rendent possible, lobservance de ces règles de conduite renforçant graduellement la coordination de plus en plus de personnes.

Tout n'est sans doute pas pour le mieux dans le meilleur des mondes dans une telle perspective, mais l'ordre économique et social qui advient ainsi n'est assurément pas le plus mauvais qui soit aux yeux de Hayek, pour qui, en matière d'économie politique, le mieux est le plus souvent l’ennemi du bien.

Départemenu de philosophie

Université du Québec à Montréal 